\title{
HEAT OF MIXING OF SOME BINARY LIQUID ALLOYS
}

\author{
B. K. Kanth* and S. K. Chakrabarti** \\ *Department of Chemistry, M.M.A.M. Campus, Tribhuvan University, Biratnagar, Nepal. \\ ** Department of Physics, M.M.A.M. Campus, Tribhuvan University, Biratnagar, Nepal.
}

\begin{abstract}
There are a large number of binary liquid alloys the thermodynamic properties of mixing of which are not symmetrical about the equi-atomic composition — deviating maximally from those of the ideal alloys. Here we have considered three such alloys —-indium-sodium, cadmium-sodium and copper-tin — and tried to calculate their heat of mixing at different concentrations of the ingredients. The liquidus lines of these alloys reveal that the constituent species form complexes. So, we have considered a quasi-lattice chemical model for computation of the thermodynamic entities. It is a statistical model in which grand partition function is used assuming that the energy of a given nearest neighbour bond is different if it belongs to the complex than if it does not. For each alloy we have started with the expression for excess free energy of mixing according to this model and computed the free energy of mixing for different concentrations of the metals within it by deriving the value of interaction parameters through successive approximation method. Thereafter, the expression for excess entropy of mixing is taken into account and the entropy of mixing is computed for different concentrations after finding out the temperature derivative of interaction parameters by the method of successive approximation. Finally the heat of mixing is calculated from these free energy of mixing and entropy of mixing on using the standard thermodynamic relation. The results explain the observed asymmetry in the heat of mixing of the said binary liquid alloys around equi-atomic composition.
\end{abstract}

Key words : Binary liquid alloy; Quasi-lattice chemical model; Heat of mixing.

\section{INTRODUCTION}

A large number of binary liquid alloys exhibit interesting behaviour as a function of concentration as regards the thermodynamic properties. The anomalous behaviour of these liquid alloys is least understood and demands extensive theoretical investigation (Bhatia and Singh 1982, Lamparter et al 1984, Prasad et al 1996, Jha et al 2001, Chakrabarti et al 2007).

The alloying behaviour of liquid alloys can be studied by the help of two distinct theories e.g. electronic theory of mixing and statistical mechanical theory of mixing. According to the first theory a liquid alloy is assumed to consist of a system of ions and electrons. The problem, usually, in this approach is tackled through pseudo-potential theory (Harrison 1966, Heine 1970, Vora et al 2002, Thakur et al 2005) and hard sphere model (Theile 1963, Lebowitz 1964, Faber 1972, Shimoji 1977). The approach cannot be used to obtain information regarding the concentration fluctuations in the long wave-length limit $\left[\mathrm{S}_{\mathrm{cc}}(0)\right]$, an important thermodynamic function which determines the stability of alloys. The other theory can be successfully used to obtain the analytical expressions for various thermodynamic functions that are not possible otherwise. The conformal solution model (Longuet-Higgins
$1951)$ has been used by many theoreticians to study $S_{c c}(0)$ of different binary alloys (Bhatia et al 1973, Alonso and March 1982). But this model cannot be used to study the shortrange-order parameters. The quasi-chemical theory (Guggenheim 1952) is successful in studying the chemical short-range-order parameters of binary liquid alloys. But such theory cannot be used to explain the asymmetry in the properties of mixing as a function of concentration.

In the present work we have considered three complex forming alloys e.g. In-Na, $\mathrm{Cd}-\mathrm{Na}$ and $\mathrm{Cu}-\mathrm{Sn}$, the thermodynamic properties of mixing of which are quite asymmetrical around the equi-atomic composition. The heat of mixing of these binary liquid alloys is taken into account and the quasi-lattice chemical model (Bhatia and Singh 1982) has been envisaged for computation of the same at different concentrations of the constituent species. It is a statistical model in which grand partition function is used assuming that the energy of a given nearest neighbour bond is different if it belongs to the complex than if it does not.

In Section 2 the calculations under this quasi-lattice chemical model are summarised to general expressions. Section 3 deals with the results. A brief conclusion is provided in Section 4 .

Author for Correspondence: S. K. Chakrabarti, Department of Physics, M. M. A. M. Campus, Tribhuvan University, Biratnagar, Nepal, Email: skc_2007@yahoo.com 


\section{FORMULATION}

The quasi-lattice chemical model for the binary liquid alloys, in essence, assumes the existence of chemical complexes $\mathrm{A}_{\mu} \mathrm{B}_{v}$, where $\mu \& v$ are small integers and $A \& B$ the constituent species of the alloy :

$$
\mu \mathrm{A}+\nu \mathrm{B} \rightleftharpoons \mathrm{A}_{\mu} \mathrm{B}_{v}
$$

According to this model the expression for excess free energy of mixing (Bhatia and Singh 1982) :

$$
\mathrm{G}_{\mathrm{M}}^{\mathrm{xs}}=\mathrm{N}\left[\mathrm{c}(1-\mathrm{c}) \omega+\Phi_{\mathrm{AB}} \Delta \omega_{\mathrm{AB}}+\Phi_{\mathrm{AA}} \Delta \omega_{\mathrm{AA}}+\Phi_{\mathrm{BB}} \Delta \omega_{\mathrm{BB}}\right],
$$

where $\mathrm{N}$ is the total number of atoms of $\mathrm{A}$ and $\mathrm{B}$ in the alloy, ' $c$ ' the concentration of A-atoms, $\omega$ 's the ordering energies and $\Phi$ 's some constants given by

$\mathrm{K}_{\mathrm{B}} \mathrm{T} \Phi_{\mu, v}=\Delta \omega_{\mathrm{AB}}[2 \beta(\mu+1, v)-2 \beta(\mu, v+1)+\beta(2 \mu-1,2 v)-\beta(2 \mu$, $2 v-1)]+\Delta \omega_{\mathrm{AA}}[\beta(2 \mu-2,2 v+1)-2 \beta(\mu, v+1)]+\Delta \omega_{\mathrm{BB}}[2 \beta(\mu+1, v)$ $-\beta(2 \mu+1,2 v-2)]$,

$\mathrm{K}_{\mathrm{B}}$ being the Boltzmann constant, $\mathrm{T}$ the absolute temperature and $\beta$ 's the usual beta functions.

Hence, the free energy of mixing of a complex forming binary liquid alloy,

$\mathrm{G}_{\mathrm{M}}=\mathrm{G}_{\mathrm{M}}^{\mathrm{xs}}+\mathrm{RT}[\mathrm{cln} \mathrm{c}+(1-\mathrm{c}) \ln (1-\mathrm{c})]$,

where $\mathrm{R}$ is the universal gas constant.

The excess entropy of mixing is given by

$$
\begin{aligned}
& \mathrm{S}_{\mathrm{M}}^{\mathrm{XS}}=-\left(\frac{\mathrm{d \textrm {G } _ { M } ^ { \mathrm { XS } }}}{\mathrm{dT}}\right)_{\mathrm{P}} \\
& =-\mathrm{N}\left[\Phi(\mathrm{c}) \frac{\mathrm{d} \omega}{\mathrm{dT}}+\Phi_{\mathrm{AB}}(\mathrm{c}) \quad\left(\Delta \omega_{\mathrm{AB}}\right)+\Phi_{\mathrm{AA}}(\mathrm{c}) \frac{\mathrm{d}}{\mathrm{dT}}\left(\Delta \omega_{\mathrm{AA}}\right)+\right. \\
& \Phi_{\mathrm{BB}}(\mathrm{c}) \frac{\mathrm{d}}{\mathrm{dT}}\left(\Delta \omega_{\mathrm{BB}}\right), \ldots \ldots \ldots \ldots \ldots . . . . . . . . . .
\end{aligned}
$$

where $\quad \Phi(\mathrm{c})=\mathrm{c}(1-\mathrm{c})$.

So, the entropy of mixing of such a binary liquid alloy,

$\mathrm{S}_{\mathrm{M}}^{\mathrm{XS}}=-\mathrm{R}[\mathrm{cln} \mathrm{C}+(1-\mathrm{c}) \ln (1-\mathrm{c})]$.

Now, the heat of mixing can be found out by using equations (iii) and (v) :

$\mathrm{H}_{\mathrm{M}}=\mathrm{G}_{\mathrm{M}}+\mathrm{TS}_{\mathrm{M}}$

\section{RESULTS AND DISCUSSION}

\subsection{Indium-Sodium Liquid Alloy}

The phase diagram of In-Na alloy suggests that the complex $\mathrm{In}_{2} \mathrm{Na}$ is most likely to be formed. So, for this alloy

$\mathrm{A} \equiv \mathrm{In}, \quad \mathrm{B} \equiv \mathrm{Na}, \quad \mu=2, \quad \nu=1$.

Finding the values of the $\beta$-functions in equation (ii), equation (i) becomes

$$
\begin{array}{r}
G_{M}^{\mathrm{xs}}=N\left[\omega c(1-c)+\Delta \omega_{A B}\left(\frac{1}{6} c+c^{2}-\frac{5}{3} c^{3}+\frac{1}{2} c^{4}\right)+\Delta \omega_{A A}\left(-\frac{1}{4} c+\frac{1}{2} c^{2}-\frac{1}{4} c^{4}\right)\right] \\
{\left[\because \operatorname{coeff}\left(\Delta \omega_{B B}\right)=0 \text { as } v<2\right]}
\end{array}
$$

The values of interaction parameters in equation (i) are determined from the experimental values of free energy of mixing of In-Na alloys at $713 \mathrm{~K}$ in the concentration range of indium from 0.1 to 0.9 (Hultgren et al 1973) by the method of successive approximation:

$$
=0.7, \quad \frac{\Delta \omega_{\mathrm{AB}}}{\mathrm{K}_{\mathrm{B}} \mathrm{T}}=-2.2, \quad \Delta \omega_{\mathrm{AA}} \approx 0 .
$$

The observed values of entropy of mixing at $713 \mathrm{~K}$ in the concentration range of indium from 0.1 to 0.9 (Hultgren et al 1973) have been used to find out the temperature derivatives of interaction parameters in equation (iv) by the successive approximation method :

$$
=6.3, \frac{1}{K_{B}} \frac{d}{d T}\left(\Delta \omega_{A B}\right)=-3.6, \frac{d}{d T}\left(\Delta \omega_{A A}\right) \approx 0, \frac{d}{d T}\left(\Delta \omega_{B B}\right) \approx 0 .
$$

With these values, finally, the heat of mixing of the indiumsodium liquid alloys at $713 \mathrm{~K}$ has been computed as a function of concentration by using equation (vi). The computed values of the heat of mixing are furnished below in Table- 1 along with its observed values in the concentration range of indium from 0.1 to 0.9 (Hultgren et al 1973).

Table 1: Heat of Mixing of In-Na liquid alloys at $713 \mathrm{~K}$.

\begin{tabular}{|c|c|c|}
\hline \multirow{2}{*}{$\mathbf{c}_{\text {In }}$} & \multicolumn{2}{|c|}{$\mathbf{H}_{\mathbf{M}} / \mathbf{R T}$} \\
\cline { 2 - 3 } & Theoretical & Experimental* \\
\hline 0.1 & -0.3788 & -0.3163 \\
\hline 0.2 & -0.7265 & -0.6510 \\
\hline 0.3 & -1.0008 & -0.9695 \\
\hline 0.4 & -1.2303 & -1.2407 \\
\hline 0.5 & -1.3889 & -1.4299 \\
\hline 0.6 & -1.4511 & -1.4638 \\
\hline 0.7 & -1.3881 & -1.2470 \\
\hline 0.8 & -1.1739 & -0.8968 \\
\hline 0.9 & -0.7670 & -0.4745 \\
\hline
\end{tabular}

*Hultgren et al, 1973

The plot of $\mathrm{H}_{\mathrm{M}} / \mathrm{RT}$ versus $\mathrm{C}_{\mathrm{In}}$ at $713 \mathrm{~K}$ is depicted in Figure1 for both the theoretical and experimental values, which show good agreement. The theoretical value of $\mathrm{H}_{\mathrm{M}}$ is minimum at $\mathrm{C}_{\mathrm{In}}=0.62$ while experimentally it is found to be minimum at $\mathrm{C}_{\mathrm{In}}=0.59$.

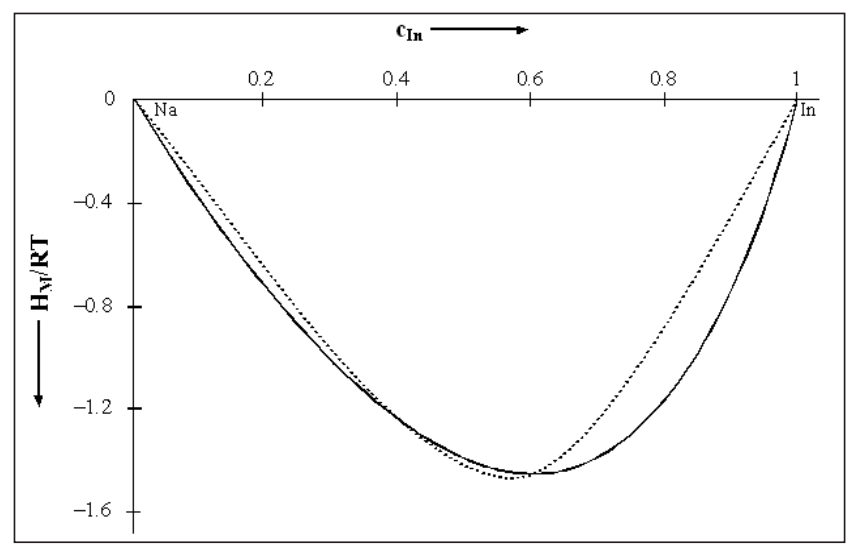

Figure 1 : Heat of mixing $\left(\mathrm{H}_{\mathrm{w}} / \mathrm{RT}\right)$ of In-Na liquid alloys at $713 \mathrm{~K}$ for different concentrations of indium. The full curve represents the theoretical values. The dotted curve shows the experimental values due to Hultgren et al (1973). 


\subsection{Cadmium-Sodium Liquid Alloy}

As revealed from the phase diagram $\mathrm{Cd}_{2} \mathrm{Na}_{\text {complex is formed }}$ within the cadmium-sodium alloy. So, for this liquid alloy

$\mathrm{A} \equiv \mathrm{Cd}, \quad \mathrm{B} \equiv \mathrm{Na}, \quad \mu=2, \quad v=1$.

Accordingly, from equations (i) and (ii) one has

$$
\begin{array}{r}
\mathrm{G}_{\mathrm{M}}^{\mathrm{xS}}=\mathrm{N}\left[\omega \mathrm{c}(1-\mathrm{c})+\Delta \omega_{\mathrm{AB}}\left(\frac{1}{6} \mathrm{c}+\mathrm{c}^{2}-\frac{5}{3} \mathrm{c}^{3}+\frac{1}{2} \mathrm{c}^{4}\right)+\Delta \omega_{\mathrm{AA}}\left(-\frac{1}{4} \mathrm{c}+\frac{1}{2} \mathrm{c}^{2}-\frac{1}{4} \mathrm{c}^{4}\right)\right] \\
{\left[\because \operatorname{coeff}\left(\Delta \omega_{\mathrm{BB}}\right)=0 \text { as } v<2\right]}
\end{array}
$$

Like before the values of interaction parameters have been found out from the experimental values of free energy of mixing at $673 \mathrm{~K}$ in the concentration range of cadmium from 0.1 to 0.9 (Hultgren et al 1973) by successive approximation method:

$$
=0.7, \quad=-2.2, \quad \Delta \omega_{\mathrm{AA}} \quad 0 .
$$

The experimental values of entropy of mixing of Cd-Na alloys at $673 \mathrm{~K}$ in the concentration range of cadmium from 0.1 to 0.9 (Hultgren et al 1973) are used, like the case of In-Na alloys, to determine the temperature derivatives of interaction parameters by the method of successive approximation :

$$
\text { = 6.3, } \quad=-3.6, \frac{\mathrm{d}}{\mathrm{dT}}\left(\Delta \omega_{\mathrm{AA}}\right) \approx 0, \frac{\mathrm{d}}{\mathrm{dT}}\left(\Delta \omega_{\mathrm{BB}}\right) \approx 0 .
$$

The heat of mixing of Cd-Na liquid alloys at $673 \mathrm{~K}$ has been computed as a function of concentration from equation (vi) on taking the above values of interaction parameters and their temperature derivatives as used for the calculation of free energy of mixing and entropy of mixing. The computed values of the heat of mixing are tabulated in Table-2 together with its observed values in the concentration range of cadmium from 0.1 to 0.9 (Hultgren et al 1973).

Table 2: Heat of Mixing of Cd-Na liquid alloys at $673 \mathrm{~K}$.

\begin{tabular}{|c|c|c|}
\hline \multirow{2}{*}{$\mathbf{c}_{\mathbf{C d}}$} & \multicolumn{2}{|c|}{$\mathbf{H}_{\mathbf{M}} / \mathbf{R T}$} \\
\cline { 2 - 3 } & Theoretical & Experimental* \\
\hline 0.1 & -0.1650 & -0.0254 \\
\hline 0.2 & -0.2288 & -0.1010 \\
\hline 0.3 & -0.3258 & -0.2177 \\
\hline 0.4 & -0.4362 & -0.3770 \\
\hline 0.5 & -0.5419 & -0.5711 \\
\hline 0.6 & -0.6218 & -0.7241 \\
\hline 0.7 & -0.6498 & -0.8236 \\
\hline 0.8 & -0.6046 & -0.7997 \\
\hline 0.9 & -0.4434 & -0.5528 \\
\hline
\end{tabular}

*Hultgren et al, 1973

The plot of $\mathrm{H}_{\mathrm{M}} / \mathrm{RT}$ versus $\mathrm{C}_{\mathrm{Cd}}$ at $673 \mathrm{~K}$ is shown in Figure-2 for both the theoretical and experimental values. Slight deviations are found in the calculated and observed values of $\mathrm{H}_{\mathrm{M}} \cdot \mathrm{H}_{\mathrm{M}}$ shows a minimum at $\mathrm{C}_{\mathrm{Cd}}=0.7$ theoretically whereas experimentally the same is at $\mathrm{c}_{\mathrm{Cd}}=0.76$.

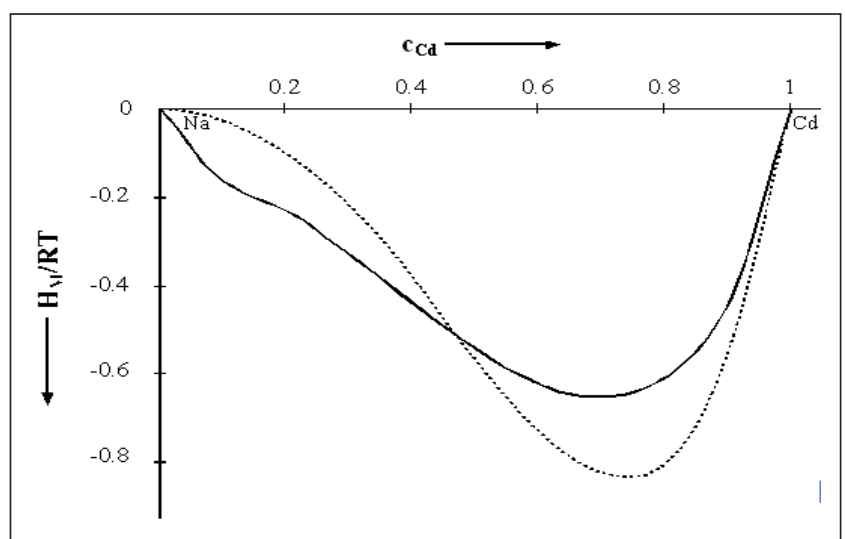

Figure 2 : Heat of mixing $\left(\mathrm{H}_{\mathrm{M}} / \mathrm{RT}\right)$ of $\mathrm{Cd}-\mathrm{Na}$ liquid alloys at $673 \mathrm{~K}$ for different concentrations of cadmium. The full curve represents the theoretical values. The dotted curve shows the experimental values due to Hultgren et al (1973).

\subsection{Copper-Tin Liquid Alloy}

Envisaging the formation of chemical complex $\mathrm{Cu}_{4} \mathrm{Sn}$ one has for the copper-tin liquid alloy :

$$
\mathrm{A} \equiv \mathrm{Cu}, \quad \mathrm{B} \equiv \mathrm{Sn}, \quad \mu=4, \quad v=1 .
$$

Finding the values of the $\beta$-functions in equation (ii), the expression for excess free energy of mixing for this alloy becomes

$$
\begin{array}{r}
\mathrm{G}_{\mathrm{M}}^{\mathrm{xs}}=\mathrm{N}\left[\omega \mathrm{c}(1-\mathrm{c})+\Delta \omega_{\mathrm{AB}}\left(\frac{27}{140} \mathrm{c}+\frac{1}{2} \mathrm{c}^{4}-\frac{4}{5} \mathrm{c}^{5}-\frac{1}{7} \mathrm{c}^{7}+\frac{1}{4} \mathrm{c}^{8}\right)+\right. \\
\left.\Delta \omega_{\mathrm{AA}}\left(-\frac{79}{840} \mathrm{c}+\frac{1}{2} \mathrm{c}^{4}-\frac{2}{5} \mathrm{c}^{5}-\frac{1}{6} \mathrm{c}^{6}+\frac{2}{7} \mathrm{c}^{7}-\frac{1}{8} \mathrm{c}^{8}\right)\right] \\
{\left[\because \operatorname{coeff}\left(\Delta \omega_{\mathrm{BB}}\right)=0 \text { as } v<2\right]}
\end{array}
$$

The interaction parameters in equation (i) are ascertained from the experimental values of free energy of mixing of $\mathrm{Cu}-$ Sn alloys at $1400 \mathrm{~K}$ in the concentration range of copper from 0.1 to 0.9 (Hultgren et al 1973) by the method of successive approximation :

$$
=-0.1, \quad=-4.2, \quad \Delta \omega_{\mathrm{AA}} \approx 0 .
$$

The observed values of entropy of mixing at $1400 \mathrm{~K}$ in the concentration range of copper from 0.1 to 0.9 (Hultgren et al 1973) are used to find out the temperature derivatives of interaction parameters in equation (iv) by the successive approximation method :

$$
=-9.2 \text {, }
$$$$
=-4.5, \frac{\mathrm{d}}{\mathrm{dT}}\left(\Delta \omega_{\mathrm{AA}}\right) \approx 0,
$$

$$
\frac{\mathrm{d}}{\mathrm{dT}}\left(\Delta \omega_{\mathrm{BB}}\right) \approx 0
$$


The computed values of the heat of mixing of $\mathrm{Cu}-\mathrm{Sn}$ liquid alloys at $1400 \mathrm{~K}$ are furnished in Table-3 along with its observed values in the concentration range of copper from 0.1 to 0.9 (Hultgren et al 1973).

Table 3: Heat of Mixing of Cu-Sn liquid alloys at $1400 \mathrm{~K}$.

\begin{tabular}{|c|c|c|}
\hline \multirow{2}{*}{$\mathbf{c}_{\mathbf{C u}}$} & \multicolumn{2}{|c|}{$\mathbf{H}_{\mathbf{M}} / \mathbf{R T}$} \\
\cline { 2 - 3 } & Theoretical & Experimental* \\
\hline 0.1 & +0.0010 & +0.0186 \\
\hline 0.2 & +0.1152 & +0.0046 \\
\hline 0.3 & +0.1688 & -0.0348 \\
\hline 0.4 & +0.1188 & -0.0948 \\
\hline 0.5 & -0.0023 & -0.1705 \\
\hline 0.6 & -0.1923 & -0.2567 \\
\hline 0.7 & -0.3812 & -0.3354 \\
\hline 0.8 & -0.4915 & -0.3515 \\
\hline 0.9 & -0.4263 & -0.2301 \\
\hline
\end{tabular}

*Hultgren et al, 1973

The plot of $\mathrm{H}_{\mathrm{M}} / \mathrm{RT}$ versus $\mathrm{C}_{\mathrm{Cu}}$ at $1400 \mathrm{~K}$ is depicted in Figure3 for both the theoretical and experimental values. It is interesting to observe that $\mathrm{H}_{\mathrm{M}}-\mathrm{C}_{\mathrm{Cu}}$ variation is clearly $\mathrm{S}$ shaped. Theoretically, for $\mathrm{C}_{\mathrm{Cu}}<0.5, \mathrm{H}_{\mathrm{M}}$ is positive and for $\mathrm{C}_{\mathrm{Cu}}>0.5, \mathrm{H}_{\mathrm{M}}$ is negative - the maximum value of the heat of mixing occurring at $\mathrm{C}_{\mathrm{Cu}}=0.3$ and the minimum value at $\mathrm{C}_{\mathrm{Cu}}=0.8$.

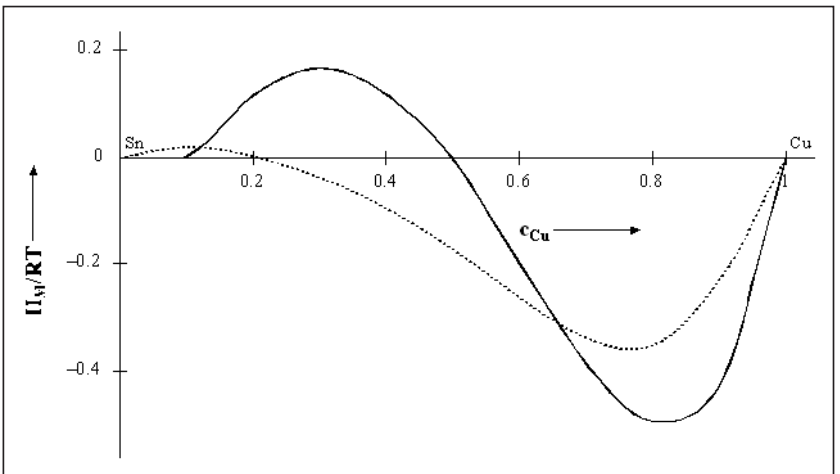

Figure 3 : Heat of mixing $\left(\mathrm{H}_{\mathrm{M}} / \mathrm{RT}\right)$ of $\mathrm{Cu}-\mathrm{Sn}$ liquid alloys at $1400 \mathrm{~K}$ for different concentrations of copper. The full curve represents the theoretical values. The dotted curve shows the experimental values due to Hultgren et al (1973).

\section{CONCLUSION}

The anomaly in the heat of mixing of In-Na, Cd-Na and Cu-Sn liquid alloys is well explained by the present theoretical model. The nature of curves as found experimentally is corroborated to a great extent by our computed values of $\mathrm{H}_{\mathrm{M}}$ for these complex forming binary liquid alloys for different concentrations of the ingredients.

\section{REFERENCES}

Alonso, J.A. and March, N.H. 1982. Physica. B114: 67.

Bhatia, A.B., Hargrove, W.H. and March, N.H. 1973. J. Phys. C6: 621. Bhatia, A.B. and Singh, R.N. 1982. Phys. Chem. Liq. 11: 285.

Bhatia, A.B. and Singh, R.N. 1982. Phys. Chem. Liq. 11: 343.

Chakrabarti, S.K., Jha, I.S., Jha, B.K. and Singh, B.P. 2007. Proc. DAE SSPS. 52: 485.

Faber, T.E. 1972. Introduction to the Theory of Liquid Metals. Cambridge Univ. Press, Cambridge.

Guggenheim, E.A. 1952. Mixtures. Oxford Univ. Press, Oxford.

Harrison, W.A. 1966. Pseudopotential in the Theory of Metals. Benjamin, New York.

Heine, V. 1970. Solid State Physics. Academic, New York.

Hultgren, R., Desai, P.D., Hawkins, D.T., Gleiser, M. and Kelley, K.K. 1973. Selected Values of the Thermodynamic Properties of Binary Alloys. A. S. M., Ohio.

Jha, N., Mishra, A.K., Rafique, S.M. and Singh, A.N. 2001. Indian J. Phys., 75A: 519.

Lamparter, P., Martin, W. and Steeb, S. 1984. J. Non-Cryst. Solids. 61: 279.

Lebowitz, J.L. 1964. Phys. Rev. AL33: 895.

Longuet-Higgins, H.C. 1951. Proc. Roy. Soc. A205: 247.

Prasad, L.C., Chatterjee, S.K. and Singh, V.N. 1996. Physica. B217: 285.

Shimoji, M. 1977. Liquid Metals. London Acad., London.

Thakur, A., Negi, N.S. and Ahluwalia, P.K. 2005. Pramana. 65: 349. Theile, E. 1963. J. Chem. Phys. Lett. 39: 474.

Vora, A.M., Patel, H.M., Gajjar, P.N. and Jain, A.R. 58. Pramana. 58: 849. 